\title{
Continuum Model for Traffic Flow considering Safe Driving Awareness Heterogeneity
}

\author{
Youzhi Zeng and Ning Zhang \\ School of Economics and Management, Beihang University, Beijing 100191, China \\ Correspondence should be addressed to Youzhi Zeng; simon.zen@163.com
}

Received 23 September 2014; Accepted 24 December 2014

Academic Editor: Luigi C. Berselli

Copyright ( 2015 Y. Zeng and N. Zhang. This is an open access article distributed under the Creative Commons Attribution License, which permits unrestricted use, distribution, and reproduction in any medium, provided the original work is properly cited.

\begin{abstract}
This paper defines the concepts of region representative vehicle and driver and region representative safe driving awareness and its heterogeneity, and, based on these concepts and a new car-following model proposed, it proposes a new continuum model for traffic flow considering region representative safe driving awareness heterogeneity. Analyses show that the new continuum model follows traffic flow anisotropy principle, and the following insights can be gotten: (1) the bigger the difference of the preceding region representative safe driving awareness coefficient minus the following region representative safe driving awareness coefficient is, the less the probability of the wrong-way travel (the negative velocity) problem in the new continuum model is; (2) when the preceding region representative safe driving awareness coefficient is not less than the following region representative safe driving awareness coefficient, there is no wrong-way travel problem in the new continuum model, and vice versa.
\end{abstract}

\section{Introduction}

The research of the continuum model for traffic flow began with LWR model, which was proposed by Lighthill and Whitham [1,2] and Richards [3], respectively. LWR model can simulate many real traffic phenomena, such as the generation and the dissipation of traffic congestion very well; however, it assumes the vehicle velocity is always in the equilibrium, so it cannot simulate the nonequilibrium traffic phenomena, such as go and stop and phantom jam. For this, some scholars put forward high-order models. The first high-order model was proposed by Payne [4]; based on the car-following model of Newell [5], Payne's model used the dynamic equation of velocity to replace the equilibrium relationship of velocity used by LWR model, and it allowed the velocity to deviate from the equilibrium relationship. Payne's model can well describe real traffic in some respects, such as go and stop and phantom jam. It does solve some drawbacks of LWR model; however, Payne's model introduces new flaws: the wrong-way travel and gas-like behavior problems (Daganzo [6]), which means in Payne's model there is a negative velocity phenomenon and the basic principle of traffic flow anisotropy that drivers only respond to frontal stimuli is violated by Payne's model. Based on Payne's model, many extended models [7-15] were proposed. For the dynamic equations of these models $[4,7-15]$ all include the density gradient term; they are all called the density gradient models. Extended models [7-15] solve some flaws of the Payne model, but they also have the wrong-way travel and gas-like behavior problems. In order to solve these problems of the density gradient model, the velocity gradient models [16-20] were proposed, which used the velocity gradient term to replace the density gradient term of the dynamic equation and solved the wrong-way travel and gas-like behavior problems.

The research of the continuum model has made great achievements, but there is no literature considering drivers' heterogeneity, such as safe driving awareness heterogeneity. However, traffic flow is a complex system composed of vehicles and drivers, and drivers' behavior has absolute effects on traffic flow stability and usually is heterogeneous, so research of drivers' heterogeneity has important theoretical and practical significance, such as the research of safe driving awareness heterogeneity. For this, this paper defines the concepts of region representative vehicle and driver and region representative safe driving awareness and its heterogeneity, and, based on these concepts and a new car-following model proposed, it proposes a new continuum model considering safe driving awareness heterogeneity. It will provide a viable 
idea to ease and inhibit traffic congestion and improve traffic flow stability.

The remainder of the paper is organized as follows. Section 2, based on the concepts of microscopic single driver's disturbance risk preference and its heterogeneity, proposes a full velocity difference model called DRPFVD model considering drivers' heterogeneity of the disturbance risk preference for car-following model, when drivers react to the relative velocity. Section 3 defines the concepts of region representative vehicle and driver and region representative safe driving awareness and its heterogeneity, and, based on these concepts and the new car-following model, it proposes a continuum model considering region representative safe driving awareness heterogeneity. Section 4 analyzes the new continuum model showing whether it meets traffic flow anisotropy and has the wrong-way problem or not and research effects of region representative safe driving awareness heterogeneity on traffic flow stability. Section 5 concludes the whole paper and puts forward a feasible method which can improve traffic flow stability and inhibit traffic congestion from macroscopic view.

\section{The Car-Following Model}

Bando et al. [21] investigated the equation of traffic dynamics based on the assumption that each vehicle had the legal velocity, which depended on the following distance from the preceding vehicle and proposed a realistic model of traffic flow. In their model the stimulus was a function of a following distance and the sensitivity was a constant and did not introduce the time lag of response. The optimal velocity model proposed by Bando et al. was called OV model, whose control equation is

$$
a_{n}(t)=a\left[V\left(\Delta x_{n}(t)\right)-v_{n}(t)\right]
$$

where $V(\cdot)$ is the optimal velocity function, which is a monotonically increasing function and has an upper bound; $\Delta x_{n}(t)=x_{n+1}(t)-x_{n}(t) ; x_{n+1}(t)$ and $x_{n}(t)$ are the positions of the preceding vehicle $n+1$ and the following vehicle $n$ at moment $t$, respectively. $a_{n}(t)$ is the acceleration of the following vehicle $n$ at moment $t, v_{n}(t)$ is the velocity of the following vehicle $n$ at moment $t$, and $a$ is the sensitive coefficient.

For the problems that OV model has, that is, too high acceleration and unrealistic deceleration and traffic collision appearing, Helbing and Tilch [22] proposed a generalized force model called GF model. Jiang et al. [23] researched the start process of traffic flow with GF model and found that the traffic flow starting wave velocity was too small, which was not consistent with real traffic. For this, they proposed a full velocity difference model called FVD model, whose control equation is

$$
a_{n}(t)=a\left[V\left(\Delta x_{n}(t)\right)-v_{n}(t)\right]+r \Delta v_{n}(t),
$$

where $r$ is drivers' sensitive coefficient of the velocity difference; $\Delta v_{n}(t)=v_{n+1}(t)-v_{n}(t)$.

So far, only Zeng et al. [24] have proposed a model called DRPOV model considering the heterogeneity of drivers' disturbance risk preference. However, they did not consider drivers' reaction to the relative velocity. For this, based on the definitions and assumptions of DRPOV model, we will propose a model considering drivers' heterogeneity of the disturbance risk preference, when drivers react to the relative velocity. The definitions and assumptions of DRPOV model are as follows.

Definition 1. $D_{t}$ is the disturbance on traffic flow happening at moment $t$. The disturbance risk preference refers to driver's reaction degree of the disturbance, which can be expressed by the disturbance risk preference coefficient. The disturbance distance is the change of the vehicle position due to $D_{t}$. The heterogeneity of the disturbance risk preference refers to different drivers making different degree reactions for the same disturbance, which is shown by the different disturbance distance. The disturbance risk preference coefficient refers to the ratio of driver's disturbance distance caused by $D_{t}$ to the moderate driver's disturbance distance caused by $D_{t}$, which can be expressed by $\beta_{n, D_{t}}(t) ; \beta_{n, D_{t}}(t)$ is a time and space variable, and an attribute of the driver $n$ itself, activated when meeting the disturbance and decided by the driver $n$ 's own factors at moment $t$, such as personal experiences and psychological factors, changing with time and space. Generally speaking, $\beta_{n, D_{t}}(t)$ changes very slowly over time, and in order to simplify the analysis and without loss of generality, assume that $\partial \beta_{n, D_{t}}(t) / \partial t=0$.

\subsection{The Hypotheses of Drivers' Heterogeneity of the Disturbance Risk Preference}

(1) According to the type of the disturbance risk preference, drivers are divided into three types: the preference, the moderate, and the aversion; the probability distribution of the number of all types is normal distribution. The mild preference, the moderate, and the mild aversion are the majority.

(2) If the driver is the preference, $\beta_{n, D_{t}}(t)>1$; if the driver is the moderate, $\beta_{n, D_{t}}(t)=1$; if the driver is the aversion, $0 \leq \beta_{n, D_{t}}(t)<1$.

Definition 2. $y_{n}^{m}(t, 1)$ is the moderate driver's disturbance distance, which can be obtained through the actual traffic investigation; the superscript $m$ means the driver is the moderate; 1 means the moderate driver's disturbance risk preference coefficient is 1 ; namely, $\beta_{n, D_{t}}^{m}(t)=1$.

$y_{n}\left(t, \beta_{n, D_{t}}(t)\right)$ is the disturbance distance of the driver whose disturbance risk preference is uncertain and it can be calculated by the formula $y_{n}\left(t, \beta_{n, D_{t}}(t)\right)=\beta_{n, D_{t}}(t) y_{n}^{m}(t, 1)$.

2.2. The Traffic Flow State Assumption. Drive on the ring road; the initial state is uniform flow; headway is $b$; the initial velocity is $V(b)$; at moment $t$, the initial position of the vehicle $n(n=1,2, \ldots, N)$ is

$$
x_{n}^{0}(t)=b n+V(b) t
$$


When traffic flow is disturbed by the disturbance $D_{t}$, the position changes into

$$
\begin{aligned}
x_{n}\left(t, \beta_{n, D_{t}}(t)\right) & =x_{n}^{0}(t)+y_{n}\left(t, \beta_{n, D_{t}}(t)\right) \\
& =x_{n}^{0}(t)+\beta_{n, D_{t}}(t) y_{n}^{m}(t, 1) \\
& =b n+V(b) t+\beta_{n, D_{t}}(t) y_{n}^{m}(t, 1) .
\end{aligned}
$$

2.3. The New Car-Following Model. Now propose a new carfollowing model considering drivers' heterogeneity of the disturbance risk preference, when drivers react to the relative velocity, which is called DRPFVD model:

$$
\begin{aligned}
a_{n}\left(t, \beta_{n, D_{t}}(t)\right)= & a\left[V\left(\Delta x_{n}\left(t, \beta_{n, D_{t}}(t)\right)\right)-v_{n}\left(t, \beta_{n, D_{t}}(t)\right)\right] \\
& +r \Delta v_{n}\left(t, \beta_{n, D_{t}}(t)\right),
\end{aligned}
$$

where

$$
\begin{aligned}
\Delta x_{n}\left(t, \beta_{n, D_{t}}(t)\right) \\
=x_{n+1}\left(t, \beta_{n+1, D_{t}}(t)\right)-x_{n}\left(t, \beta_{n, D_{t}}(t)\right) \\
=b+\Delta y_{n}\left(t, \beta_{n, D_{t}}(t)\right) \\
=b+\beta_{n+1, D_{t}}(t) y_{n+1}^{m}(t, 1)-\beta_{n, D_{t}}(t) y_{n}^{m}(t, 1) .
\end{aligned}
$$

DRPFVD model considers drivers' heterogeneity of the disturbance risk preference, when drivers react to the relative velocity, and therefore DRPFVD model is more consistent with real traffic.

\section{A New Continuum Model}

Based on the above new car-following model and definitions and assumptions, this paper proposes a new continuum traffic flow model considering safe driving awareness heterogeneity. Before proposing the new model, make the following definitions and assumptions firstly.

3.1. The Hypotheses of Region Average Traffic Condition. In order to transfer the microscopic discrete variables into the macroscopic continuum variables, just like Jiang et al. [18], assume that the dynamic properties of vehicle $n$ at position $x$ at moment $t$ represent the average traffic condition in the following region: $[x-(1 / 2) \Delta, x+(1 / 2) \Delta]$ decided by the dynamic properties of vehicle $n+1$ at position $x+\Delta$ at moment $t$ which represents the average traffic condition in the preceding region $[x+(1 / 2) \Delta, x+(3 / 2) \Delta] ; \Delta$ is the intervehicle space between the following region representative vehicle $n$ and the preceding region representative vehicle $n+1$ and corresponds to $\Delta x$ in car-following model.

Definition 3. Region representative vehicle of $[x-(1 / 2) \Delta, x+$ $(1 / 2) \Delta]$ is vehicle $n$; region representative driver of $[x-$ $(1 / 2) \Delta, x+(1 / 2) \Delta]$ is diver $n$; region representative safe driving awareness refers to region representative driver's disturbance reaction degree, which can be expressed by the region representative safe driving awareness coefficient.
Region representative safe driving awareness heterogeneity refers to different region representative drivers making different degree reactions to the same disturbance, which is shown by the different disturbance distance. $\beta_{D_{t}}(x, t)$ is the region representative disturbance risk preference coefficient and $\delta_{D_{t}}(x, t)$ is the region representative safe driving awareness coefficient of $[x-(1 / 2) \Delta, x+(1 / 2) \Delta] ; \beta_{D_{t}}(x+\Delta, t)$ is the region representative disturbance risk preference coefficient, $\delta_{D_{t}}(x+\Delta, t)$ is the region representative safe driving awareness coefficient of $[x+(1 / 2) \Delta, x+(3 / 2) \Delta]$, and so on. The disturbance risk preference is an important performance of safe driving awareness, and the lower the former is, the higher the latter is, and vice versa, so we can assume that $\beta_{D_{t}}(x, t)=$ $k / \delta_{D_{t}}(x, t), k>0$. For the sake of simplicity and without loss of generality, assume that $\beta_{D_{t}}(x, t)=1 / \delta_{D_{t}}(x, t)$ and therefore $\Delta \delta_{D_{t}}(x, t)$ and $\Delta \beta_{D_{t}}(x, t)$ have the opposite sign because of

$$
\begin{aligned}
\Delta \beta_{D_{t}}(x, t) & =\beta_{D_{t}}(x+\Delta, t)-\beta_{D_{t}}(x, t) \\
& =\frac{\delta_{D_{t}}(x, t)-\delta_{D_{t}}(x+\Delta, t)}{\delta_{D_{t}}(x+\Delta, t) \delta_{D_{t}}(x, t)} \\
& =-\frac{\Delta \delta_{D_{t}}(x, t)}{\delta_{D_{t}}(x+\Delta, t) \delta_{D_{t}}(x, t)} .
\end{aligned}
$$

Define $\Delta \delta_{D_{t}}(x, t) / \delta_{D_{t}}(x+\Delta, t) \delta_{D_{t}}(x, t)$ as the relative difference of the preceding region representative safe driving awareness coefficient minus the following region representative safe driving awareness coefficient, which is also the relative size of both coefficients. $\beta_{D_{t}}(x, t)$ and $\delta_{D_{t}}(x, t)$ are the time and space variables, and an attribute of region representative driver $n$ itself, activated when meeting the disturbance and decided by the region representative driver $n$ 's own factors at position $x$ at moment $t$, such as personal experiences and psychological factors, changing with time and space. Generally speaking, $\beta_{D_{t}}(x, t)$ and $\delta_{D_{t}}(x, t)$ change very slowly over time, and in order to simplify the analysis and without loss of generality, assume that $\partial \beta_{D_{t}}(x, t) / \partial t=0$ and $\partial \delta_{D_{t}}(x, t) / \partial t=0$.

\subsection{The Hypotheses of Region Representative Safe Driving Awareness}

(1) According to the type of the safe driving awareness, the region representative driver is divided into three types: the low safe driving awareness, the moderate safe driving awareness, and the high safe driving awareness; the probability distribution of the number of all types is normal distribution and the region representative drivers who have the mild low safe driving awareness, the moderate safe driving awareness and the mild high safe driving awareness are in the majority.

(2) In the region $[x-(1 / 2) \Delta, x+(1 / 2) \Delta]$, if the region representative driver has the low safe driving awareness, $0 \leq \delta_{D_{t}}(x, t)<1$; if the region representative driver has the moderate safe driving awareness, $\delta_{D_{t}}(x, t)=$ 1 ; if the region representative driver has the high safe driving awareness, $\delta_{D_{t}}(x, t)>1$. 
The effects of the specific probability distribution of the number of all types and the more specific value of $\delta_{D_{t}}(x, t)$ will be further researched.

Then we can transfer the microscopic discrete variables into the macroscopic continuum variables as follows:

$$
\begin{gathered}
\beta_{n, D_{t}}(t) \longrightarrow \beta_{D_{t}}(x, t)=\frac{1}{\delta_{D_{t}}(x, t)} \\
\beta_{n+1, D_{t}}(t) \longrightarrow \beta_{D_{t}}(x+\Delta, t)=\frac{1}{\delta_{D_{t}}(x+\Delta, t)} \\
v_{n}\left(t, \beta_{n, D_{t}}(t)\right) \longrightarrow u\left(x, t, \beta_{D_{t}}(x, t)\right) \\
v_{n+1}\left(t, \beta_{n+1, D_{t}}(t)\right) \longrightarrow u\left(x+\Delta, t, \beta_{D_{t}}(x+\Delta, t)\right) \\
V\left(\Delta x_{n}\left(t, \beta_{n, D_{t}}(t)\right)\right) \longrightarrow u_{e}\left(\rho\left(x, t, \beta_{D_{t}}(x, t)\right)\right) \\
a=\frac{1}{\tau}, \quad r=\frac{1}{T}, \\
\beta_{n, D_{t}}^{m}(t)=1 \longrightarrow \beta_{D_{t}}^{m}(x, t)=1, \\
y_{n}^{m}(t, 1) \longrightarrow y^{m}(x, t, 1), \\
y_{n}\left(t, \beta_{n, D_{t}}(t)\right)=\beta_{n, D_{t}}(t) y_{n}^{m}(t, 1) \longrightarrow y\left(x, t, \beta_{D_{t}}(x, t)\right) \\
=\beta_{D_{t}}(x, t) y^{m}(x, t, 1)
\end{gathered}
$$

where $u$ is the average velocity, $u_{e}(\rho)$ is the equilibrium velocity, $\tau$ is the relaxation time, and $T$ is the reaction delay time. Substituting (8a)-(8d) into (5) of the new carfollowing model, we can get the dynamic equation of the new continuum model considering region representative safe driving awareness heterogeneity:

$$
\begin{gathered}
\frac{d u\left(x, t, \beta_{D_{t}}(x, t)\right)}{d t} \\
=\frac{u_{e}(\rho)-u\left(x, t, \beta_{D_{t}}(x, t)\right)}{\tau} \\
+r\left[u\left(x+\Delta, t, \beta_{D_{t}}(x+\Delta, t)\right)-u\left(x, t, \beta_{D_{t}}(x, t)\right)\right] \\
\quad \beta_{D_{t}}(x+\Delta, t)=\beta_{D_{t}}(x, t)+\Delta \beta_{D_{t}}(x, t) .
\end{gathered}
$$

Substituting (10) into (9) and carrying out the Taylor series expansion for the right side of (9) and neglecting the highorder terms, we have

$$
\begin{aligned}
& \frac{d u\left(x, t, \beta_{D_{t}}(x, t)\right)}{d t} \\
& =\frac{u_{e}(\rho)-u\left(x, t, \beta_{D_{t}}(x, t)\right)}{\tau}+r \Delta \frac{\partial u\left(x, t, \beta_{D_{t}}(x, t)\right)}{\partial x} \\
& \quad+r\left(\Delta \beta_{D_{t}}(x, t)\right) \frac{\partial u\left(x, t, \beta_{D_{t}}(x, t)\right)}{\partial \beta_{D_{t}}(x, t)} .
\end{aligned}
$$

Assume that $r \Delta=\Delta / T=c_{0}$, which is the propagation speed of small disturbance. Equation (11) can be rewritten as

$$
\begin{aligned}
& \frac{\partial u}{\partial t} \cdot \frac{\partial t}{\partial t}+\frac{\partial u}{\partial x} \cdot \frac{\partial x}{\partial t}+\frac{\partial u}{\partial \beta_{D_{t}}(x, t)} \cdot \frac{\partial \beta_{D_{t}}(x, t)}{\partial t} \\
& \quad=c_{0} \cdot \frac{\partial u}{\partial x}+\frac{u_{e}-u}{\tau}+r\left(\Delta \beta_{D_{t}}(x, t)\right) \frac{\partial u}{\partial \beta_{D_{t}}(x, t)}
\end{aligned}
$$

According to Definition 3,

$$
\frac{\partial \beta_{D_{t}}(x, t)}{\partial t}=0 .
$$

Then, (12) can be rewritten as

$$
\begin{aligned}
& \frac{\partial u}{\partial t}+u \frac{\partial u}{\partial x}=c_{0} \cdot \frac{\partial u}{\partial x}+\frac{u_{e}-u}{\tau}+r\left(\Delta \beta_{D_{t}}(x, t)\right) \frac{\partial u}{\partial \beta_{D_{t}}(x, t)} \\
& \beta_{D_{t}}(x, t)=\frac{1}{\delta_{D_{t}}(x, t)}, \quad \beta_{D_{t}}(x+\Delta, t)=\frac{1}{\delta_{D_{t}}(x+\Delta, t)}
\end{aligned}
$$

which is the dynamic equation of the new continuum model considering region representative safe driving awareness heterogeneity. Combining the conservation equation of LWR model, we can get the equations of the new continuum model:

$$
\begin{gathered}
\frac{\partial \rho(x, t)}{\partial t}+\frac{\partial q(x, t)}{\partial x}=\frac{\partial \rho(x, t)}{\partial t}+\frac{\partial \rho(x, t) u(x, t)}{\partial x}=s(x, t), \\
\frac{\partial u}{\partial t}+u \frac{\partial u}{\partial x}=c_{0} \cdot \frac{\partial u}{\partial x}+\frac{u_{e}-u}{\tau}+r\left(\Delta \beta_{D_{t}}(x, t)\right) \frac{\partial u}{\partial \beta_{D_{t}}(x, t)} \\
\beta_{D_{t}}(x, t)=\frac{1}{\delta_{D_{t}}(x, t)}, \quad \beta_{D_{t}}(x+\Delta, t)=\frac{1}{\delta_{D_{t}}(x+\Delta, t)},
\end{gathered}
$$

where $x, t$ are the space variable and the time variable, respectively; $\rho(x, t)$ is traffic density, $q(x, t)$ is traffic flow, and $s(x, t)$ is volume of traffic that enters the road (a negative value for exiting traffic). From (15b), we can see that the macroscopic acceleration is independent of the absolute size of region representative drivers' safe driving awareness coefficient and depends on the relative difference of the preceding region representative driver's safe driving awareness coefficient minus the following region representative driver's safe driving awareness coefficient, which is also the relative size of both coefficients. 


\section{The Anisotropic Property and Wrong-Way Travel Analysis}

The matrix expression of the continuum model of this paper is

$$
\begin{aligned}
& {\left[\begin{array}{l}
\frac{\partial \rho}{\partial t} \\
\frac{\partial u}{\partial t}
\end{array}\right]+\left[\begin{array}{cc}
u & \rho \\
0 & u-c_{0}
\end{array}\right] \cdot\left[\begin{array}{l}
\frac{\partial \rho}{\partial x} \\
\frac{\partial u}{\partial x}
\end{array}\right]} \\
& \quad=\left[\frac{u_{e}-u}{\tau}+r \Delta \beta_{D_{t}}(x, t) \frac{\partial u}{\partial \beta_{D_{t}}(x, t)}\right] .
\end{aligned}
$$

The eigenvalues $\lambda$ of the matrix $A=\left[\begin{array}{cc}u & \rho \\ 0 & u-c_{0}\end{array}\right]$ are gotten by setting

$$
\operatorname{det}[A-\lambda E]=\left|\begin{array}{cc}
u-\lambda & \rho \\
0 & u-c_{0}-\lambda
\end{array}\right|=0
$$

where $E$ is identity matrix. There are the characteristic velocities of the new continuum model, that is,

$$
\lambda_{1}=\left(\frac{d x}{d t}\right)_{1}=u, \quad \lambda_{2}=\left(\frac{d x}{d t}\right)_{2}=u-c_{0} .
$$

For $c_{0}>0,(18)$ means the new continuum model does not have any characteristic velocity greater than the macroscopic traffic flow velocity, which means traffic flow anisotropic principle is obeyed by the new continuum model; namely, driver only responds to frontal stimuli in the new continuum model.

Next, we will discuss whether there is negative travel velocity, namely, wrong-way travel problem in the new continuum model. The initial conditions given by Daganzo [6] are

$$
\begin{gathered}
u=0, \quad \rho=\rho_{m} H(x), \quad \forall x \leq A(A>0), t=0, \\
u=0, \quad x=A, \quad t>0,
\end{gathered}
$$

where $H(x)$ is the Heaviside function and $H(x)=\{0, x \leq$ $0 ; 1, x>0\} . \rho_{m}$ is the jam density. In this case, traffic flow will have no change, which is the correct equilibrium solution to this problem, which means

$$
u=0, \quad \Delta u=0 .
$$

Substituting (20) into (15b), we can get $u_{e}=0$, which means there is no negative velocity, namely, wrong-way travel problem in the new continuum model in this case.

For more general scenario, assuming at a certain position $u=0$, from (15b), we can know that the acceleration at this position is

$$
\frac{d u}{d t}=c_{0} \cdot \frac{\partial u}{\partial x}+\frac{u_{e}}{\tau}+r \Delta \beta_{D_{t}}(x, t) \frac{\partial u}{\partial \beta_{D_{t}}(x, t)} .
$$

As pointed out by Jiang et al. [18], $c_{0} \cdot(\partial u / \partial x)+u_{e} / \tau \geq 0$. Combining $r>0$, in (21), in order to judge whether the acceleration $d u / d t$ is negative or not, what we need to do is to discuss the sign of $\partial u / \partial \beta_{D_{t}}(x, t)$. According to DRPFVD model (5) and (6), when other parameters values are given, there is a microscopic effect path:

$$
\begin{aligned}
\beta_{n, D_{t}}(t) \uparrow / \downarrow & \longrightarrow \Delta x_{n}\left(t, \beta_{n, D_{t}}(t)\right) \downarrow / \uparrow \\
& \longrightarrow V\left(\Delta x_{n}\left(t, \beta_{n, D_{t}}(t)\right)\right) \downarrow / \uparrow \\
& \longrightarrow a_{n}\left(t, \beta_{n, D_{t}}(t)\right) \downarrow / \uparrow \\
& \longrightarrow v_{n}\left(t, \beta_{n, D_{t}}(t)\right) \downarrow / \uparrow
\end{aligned}
$$

and therefore there is a macroscopic effect path by the corresponding relation between macroscopic variables and microscopic variables:

$$
\begin{aligned}
\beta_{D_{t}}(x, t) \uparrow / \downarrow & \longrightarrow \rho\left(x, t, \beta_{D_{t}}(x, t)\right) \uparrow / \downarrow \\
& \longrightarrow u_{e}\left(\rho\left(x, t, \beta_{D_{t}}(x, t)\right)\right) \downarrow / \uparrow \\
& \longrightarrow \frac{d u\left(x, t, \beta_{D_{t}}(x, t)\right)}{d t} \downarrow / \uparrow \\
& \longrightarrow u\left(x, t, \beta_{D_{t}}(x, t)\right) \downarrow / \uparrow
\end{aligned}
$$

which means $\partial u / \partial \beta_{D_{t}}(x, t)<0$, and, therefore, the following conclusions can be gotten:

(1) when $\Delta \beta_{D_{t}}(x, t)>0$, namely, $\delta_{D_{t}}(x, t)>\delta_{D_{t}}(x+$ $\Delta, t)$, in this case, $r \Delta \beta_{D_{t}}(x, t)\left(\partial u / \partial \beta_{D_{t}}(x, t)\right)<0$ and according to (21), when $\left|r \Delta \beta_{D_{t}}(x, t)\left(\partial u / \partial \beta_{D_{t}}(x, t)\right)\right|$ is big enough, $d u / d t<0$ which means there is wrongway travel problem in new continuum model when $\Delta \delta_{D_{t}}(x, t)<0$

(2) when $\Delta \beta_{D_{t}}(x, t)=0$, namely, $\delta_{D_{t}}(x, t)=\delta_{D_{t}}(x+\Delta, t)$, in this case, substituting $\Delta \beta_{D_{t}}(x, t)=0$ into (21) and using $c_{0} \cdot(\partial u / \partial x)+u_{e} / \tau \geq 0$ (Jiang et al. [18]), we have $d u / d t \geq 0$, which means there is no wrong-way travel problem in new continuum model when $\Delta \delta_{D_{t}}(x, t)=$ 0 .

(3) when $\Delta \beta_{D_{t}}(x, t)<0$, namely, $\delta_{D_{t}}(x, t)<\delta_{D_{t}}(x+$ $\Delta x, t)$, in this case, $r \Delta \beta_{D_{t}}(x, t)\left(\partial u / \partial \beta_{D_{t}}(x, t)\right)>0$, $d u / d t>0$, which means there is no wrong-way travel problem in new continuum model when $\Delta \delta_{D_{t}}(x, t)>$ 0 .

According to (15b) and $\partial u / \partial \beta_{D_{t}}(x, t)<0, r>0$, and therefore $\partial(d u / d t) / \partial \Delta \beta_{D_{t}}(x, t)=r\left(\partial u / \partial \beta_{D_{t}}(x, t)\right)<0$ which means that the smaller $\Delta \beta_{D_{t}}(x, t)$ namely the bigger $\Delta \delta_{D_{t}}(x, t)$, the bigger the accelerate $d u / d t$ and the less the probability of wrong-way travel (the negative velocity) problem in the new continuum model.

From what has been discussed above, the following insights can be gotten: the bigger $\Delta \delta_{D_{t}}(x, t)$ is, the less probability of wrong-way travel problem in the new continuum model is and the better traffic flow stability is; when $\Delta \delta_{D_{t}}(x, t) \geq 0$, there is no wrong-way travel problem in the new continuum model, and when $\Delta \delta_{D_{t}}(x, t)<0$, there is the wrong-way problem in the new continuum model. 


\section{Conclusions}

The traditional continuum model for traffic flow works rather well under many circumstances and can explain many real traffic phenomena well; however, a common deficiency of these models is that they have not considered a real traffic fact, that is, the drivers' heterogeneity, such as safe driving awareness heterogeneity. For this, this paper defines the concepts of region representative vehicle and driver and region representative safe driving awareness and its heterogeneity, and, based on these concepts and a new car-following model, it proposes a new continuum model considering region representative safe driving awareness heterogeneity. Analyses show that the new continuum model follows traffic flow anisotropy principle, and the following insights can be gotten: the bigger the difference of the preceding region representative safe driving awareness coefficient minus the following region representative safe driving awareness coefficient is, the less the probability of wrong-way travel problem in the new continuum model is and the better the traffic flow stability is, and when the preceding region representative safe driving awareness coefficient is not less than the following region representative safe driving awareness coefficient, there is no wrong-way travel problem in the new continuum model, and vice versa. It provides a viable idea to improve traffic flow stability and inhibit traffic congestion. For example, strengthen all social drivers' safe driving awareness education to increase drivers' safe driving awareness.

\section{Conflict of Interests}

The authors declare that there is no conflict of interests regarding the publication of this paper.

\section{Acknowledgment}

This work is supported by the National Natural Science Foundation of China (Grant no. 70971003).

\section{References}

[1] M. J. Lighthill and G. B. Whitham, "On kinematic waves. I. Flood movement in long rivers," Proceedings of the Royal Society London Series A, vol. 229, no. 1178, pp. 281-316, 1955.

[2] M. J. Lighthill and G. B. Whitham, "On kinematic waves: II. A theory of traffic flow on long crowded roads," Proceedings of the Royal Society of London A, vol. 229, no. 1178, pp. 317-345, 1995.

[3] P. I. Richards, "Shock waves on the highway," Operations Research, vol. 4, no. 1, pp. 42-51, 1956.

[4] H. J. Payne, "Models of freeway traffic and control," Mathematical Models of Public Systems, vol. 1, pp. 51-61, 1971.

[5] G. F. Newell, "Nonlinear effects in the dynamics of car following," Operations Research, vol. 9, pp. 209-229, 1961.

[6] C. F. Daganzo, "Requiem for second-order fluid approximations of traffic flow," Transportation Research Part B, vol. 29, no. 4, pp. 277-286, 1995.

[7] M. Papageorgiou, "A hierarchical control system for freeway traffic," Transportation Research Part B, vol. 17, no. 3, pp. 251261, 1983.
[8] R. D. Kühne, "Macroscopic freeway model for dense trafficstop-start waves and incident direction," in Proceedings of the 9th International Symposium on Transportation and Traffic Theory, pp. 20-42, 1984.

[9] P. G. Michalopoulos, P. Yi, and A. S. Lyrintzis, "Continuum modelling of traffic dynamics for congested freeways," Transportation Research Part B, vol. 27, no. 4, pp. 315-332, 1993.

[10] B. S. Kerner and P. Konhäuser, "Cluster effect in initially homogeneous traffic flow," Physical Review E, vol. 48, no. 4, pp. R2335-R2338, 1993.

[11] B. S. Kerner and P. Konhäuser, "Structure and parameters of clusters in traffic flow," Physical Review E, vol. 50, no. 1, pp. 5483, 1994.

[12] H. Y. Lee, H. W. Lee, and D. Kim, "Origin of synchronized traffic flow on highways and its dynamic phase transitions," Physical Review Letters, vol. 81, no. 5, pp. 1130-1133, 1998.

[13] H. Y. Lee, H.-W. Lee, and D. Kim, "Dynamic states of a continuum traffic equation with on-ramp," Physical Review E, vol. 59, no. 5, pp. 5101-5111, 1999.

[14] H. M. Zhang, "A theory of nonequilibrium traffic flow," Transportation Research Part B: Methodological, vol. 32, no. 7, pp. 485498, 1998.

[15] P. Berg and A. Woods, "Traveling waves in an optimal velocity model of freeway traffic," Physical Review E, vol. 63, no. 3, part 2, Article ID 036107, 2001.

[16] A. Aw and M. Rascle, "Resurrection of "second order" models of traffic flow," SIAM Journal on Applied Mathematics, vol. 60, no. 3, pp. 916-938, 2000.

[17] H. M. Zhang, "A non-equilibrium traffic model devoid of gaslike behavior," Transportation Research Part B, vol. 36, no. 3, pp. 275-290, 2002.

[18] R. Jiang, Q. S. Wu, and Z.-J. Zhu, "A new continuum model for traffic flow and numerical tests," Transportation Research Part B: Methodological, vol. 36, no. 5, pp. 405-419, 2002.

[19] R. Jiang, Q. Wu, and Z. Zhu, "A new dynamics model for traffic flow," Chinese Science Bulletin, vol. 46, no. 4, pp. 345-349, 2001.

[20] Y. Xue and S. Q. Dai, "Continuum traffic model with the consideration of two delay time scales," Physical Review EStatistical, Nonlinear, and Soft Matter Physics, vol. 68, no. 6, Article ID 066123, 2003.

[21] M. Bando, K. Hasebe, A. Nakayama, A. Shibata, and Y. Sugiyama, "Dynamical model of traffic congestion and numerical simulation," Physical Review E, vol. 51, no. 2, pp. 1035-1042, 1995.

[22] D. Helbing and B. Tilch, "Generalized force model of traffic dynamics," Physical Review E: Statistical Physics, Plasmas, Fluids, and Related Interdisciplinary Topics, vol. 58, no. 1, pp. 133138, 1998.

[23] R. Jiang, Q. Wu, and Z. Zhu, "Full velocity difference model for a car-following theory," Physical Review E, vol. 64, no. 1, Article ID 017101, 2001.

[24] Y.-Z. Zeng, N. Zhang, and L.-J. Liu, "A new car-following model considering drivers' heterogeneity of the disturbance risk appetite," Acta Physica Sinica, vol. 63, no. 6, Article ID 068901, 2014. 


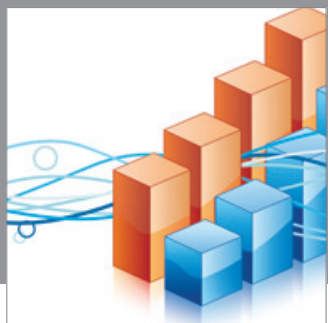

Advances in

Operations Research

mansans

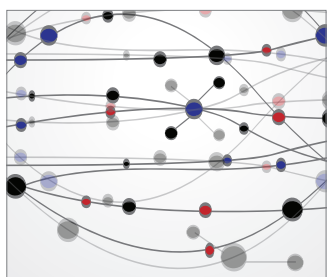

The Scientific World Journal
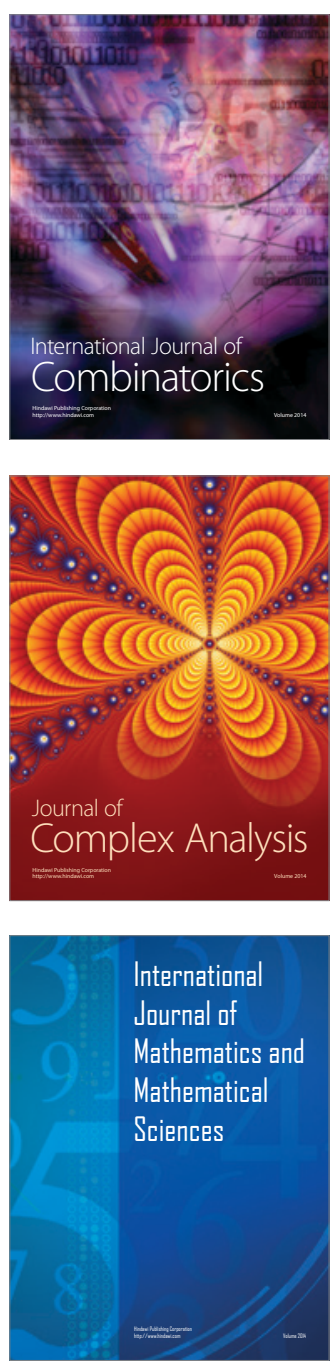
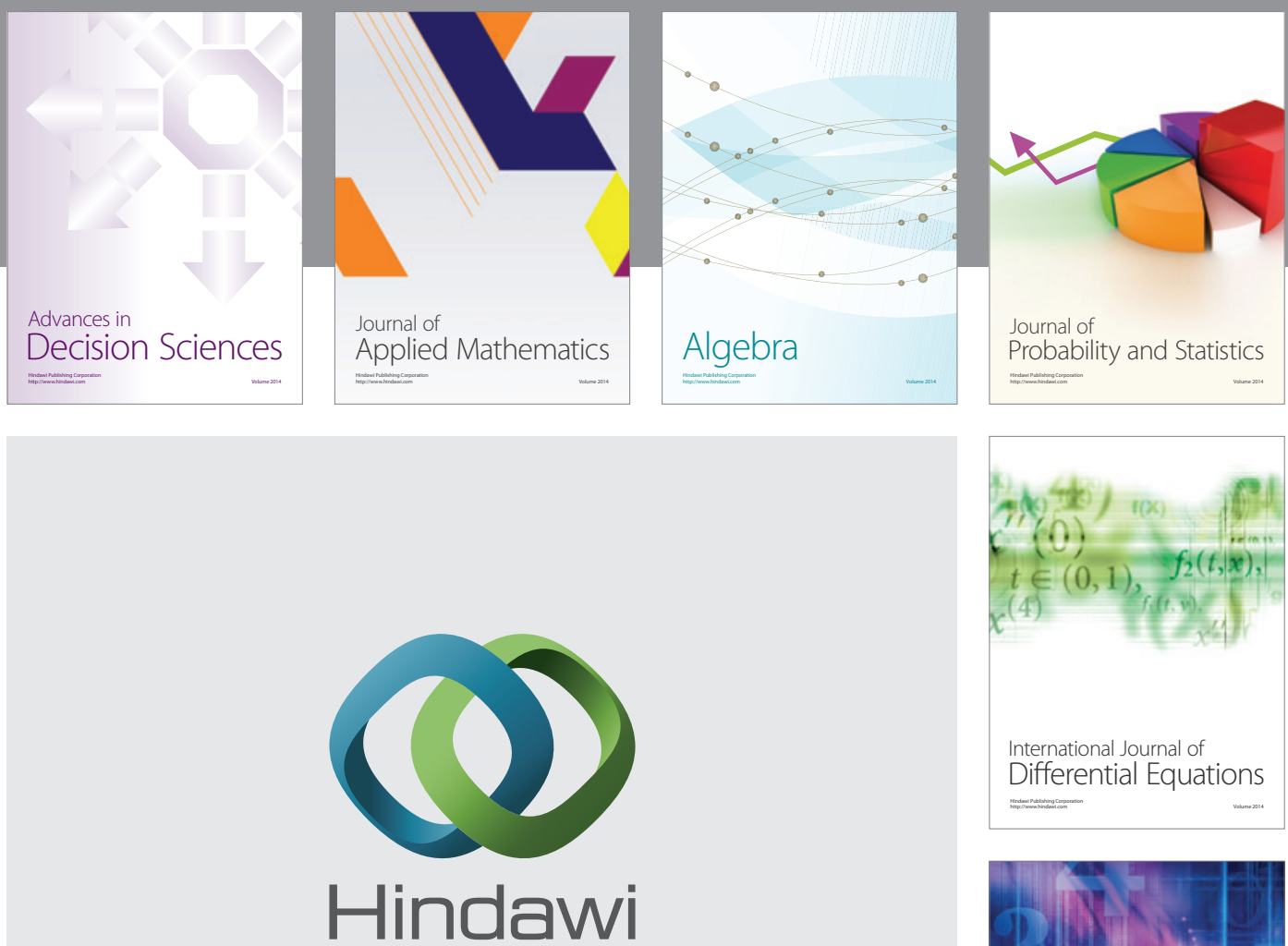

Submit your manuscripts at http://www.hindawi.com
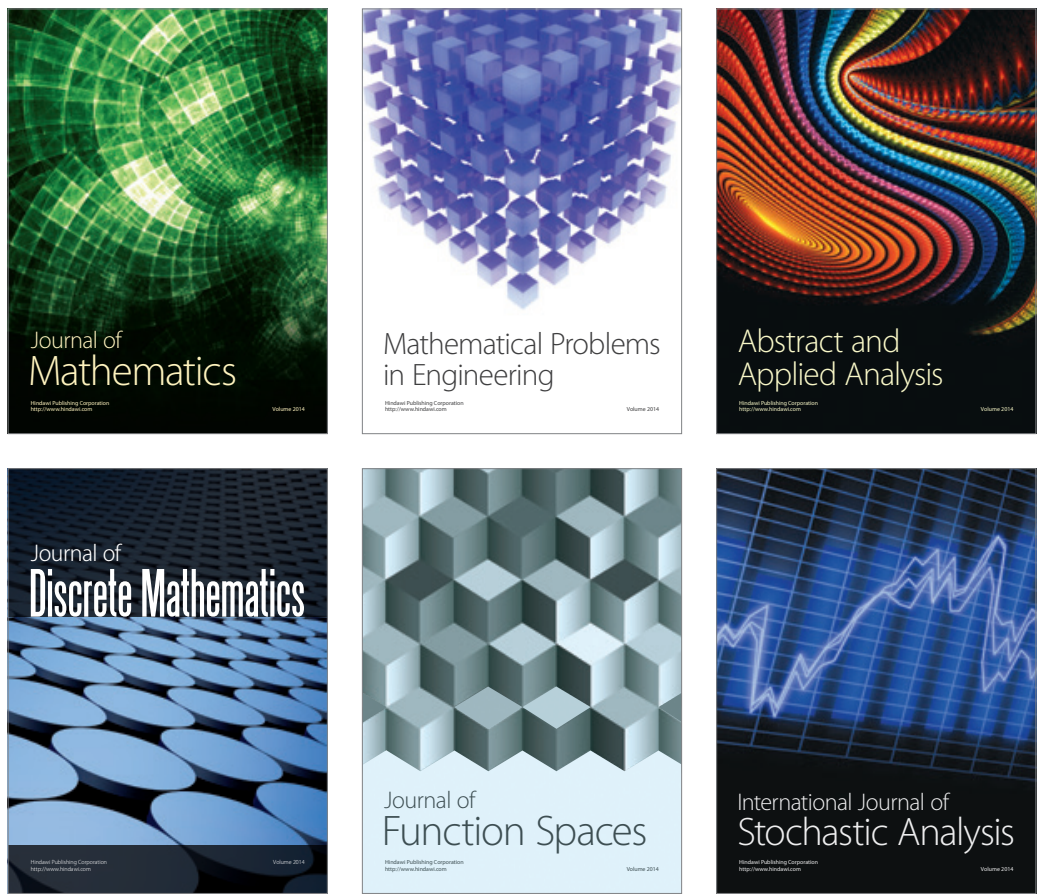

Journal of

Function Spaces

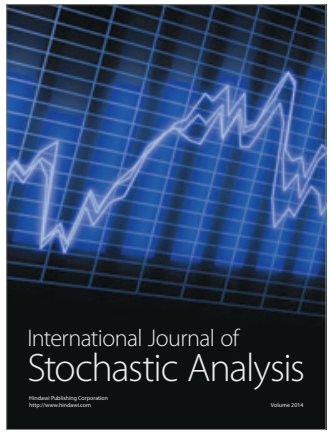

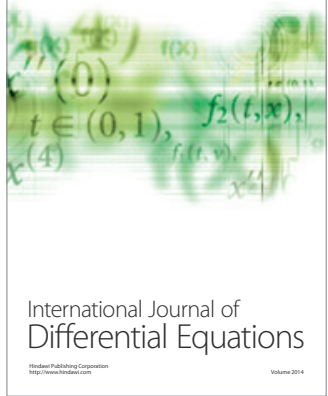
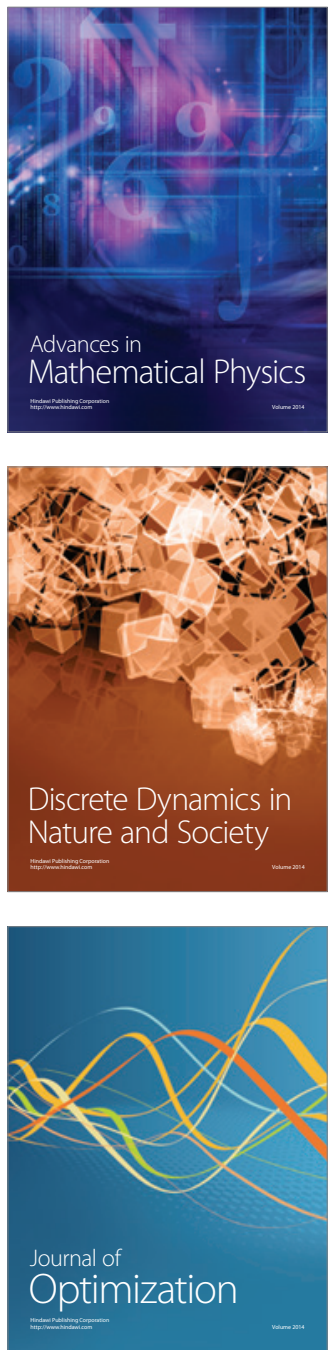